\title{
Photo-Induced Reduction Reaction of Methylene Blue in an Ionic Liquid
}

\author{
Jun-ichi Kadokawa, Hironori Izawa, Tomoya Ohta, Satoshi Wakizono, Kazuya Yamamoto \\ Graduate School of Science and Engineering, Kagoshima University, Kagoshima, Japan \\ E-mail: kadokawa@eng.kagoshima-u.ac.jp \\ Received September 24, 2011; revised October 31, 2011; accepted November 9, 2011
}

\begin{abstract}
Reduction of methylene blue (MB) occurred by photo irradiation at $280-370 \mathrm{~nm}$ wavelengths to a solution of $\mathrm{MB}$ in an ionic liquid, 1-butyl-3-methylimidazolium chloride (BMIMCl), which was confirmed by color change and UV-Vis measurement of the solution. Furthermore, the reduced MB was oxidized again by standing the solution under the conditions of light shielding at $50^{\circ} \mathrm{C}$. The fluorescence spectra of the solution excited at $350 \mathrm{~nm}$ suggested that the photo-induced reduction probably took place via electron-transfer from $\mathrm{BMIMCl}$ to $\mathrm{MB}$.
\end{abstract}

Keywords: Methylene Blue, Ionic Liquid, Reduction, Photo Irradiation

\section{Introduction}

Redox dyes where color depends on oxidation state have been useful in studies in conjugation with electrochemical applications such as a redox indicator [1]. It can be expected that development of new redox system using redox dyes leads to further application of the dyes. Methylene blue (MB) is one of the representative redox dyes, which possesses good electrochemical properties via its redox reactions [2-6]. Therefore, it has been widely used for basic electrochemical studies and applications, for example, electrocatalysis, solar cells, and biosensors $[2,7,8]$. Redox reactions of MB reversibly occurred according to Scheme 1. An oxidized MB exhibits blue color, whereas a reduced MB (leucomethylene blue) has colorless nature.

On the other hand, ionic liquids, which are low-melting salts and form liquids at the temperature below the boiling point of water, have continued to receive a great deal of attention for a wide variety of possible applications, such as the solvent system for a large number of organic and inorganic reactions, gel components, catalysis, sepa-

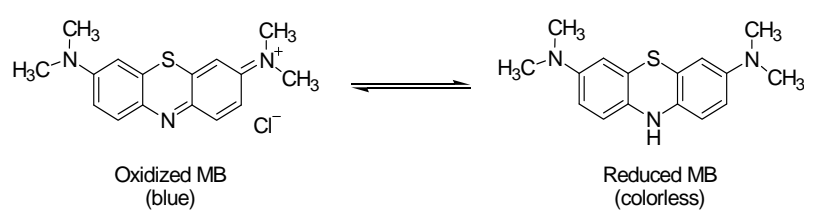

Scheme 1. Redox reactions of $\mathrm{MB}$. ration, and electrochemical studies [9-17]. The utility of the ionic liquids as media for various photophysical and photochemical studies has also been explored [18-20]. For example, photo-induced electron-transfer reactions in ionic liquids have been reported [21-23]. In this paper, we report photo-induced reduction reaction of MB in an imidazolium-type ionic liquid, 1-butyl-3-methylimidazolium chloride (BMIMCl) in the absence of additional reductant. Furthermore, the reduced MB could be oxidized again by molecular oxygen under the conditions of light shielding. Previously, redox reactions of some substrates by pulse radiolysis in ionic liquids have also been reported $[24,25]$.

\section{Results and Discussion}

When the photo irradiation at $280-370 \mathrm{~nm}$ wavelengths to a solution of MB in BMIMCl $(0.125 \mu \mathrm{mol} / \mathrm{g})$ was performed at room temperature for $12 \mathrm{~h}$, decolorization of the solution occurred. We assumed that this decolorization was owing to the reduction of $\mathrm{MB}$, because it has been well-known that the similar decolorization happens by the general reduction reaction of $\mathrm{MB}$ in the presence of some reductants such as glucose. To confirm occurrence of the reduction of $\mathrm{MB}$ in BMIMCl by the photo irradiation, the UV-Vis measurement of the resulting solution after the irradiation for $12 \mathrm{~h}$ was conducted. In Figure 1, the UV-Vis spectra of the solution of MB in BMIMCl before and after the photo irradiation are shown. 
The absorption at around $660 \mathrm{~nm}$ due to MB obviously decreased by the photo irradiation to largely disappear after $12 \mathrm{~h}$. When the decolorized solution was left standing under the conditions of light shielding at $50^{\circ} \mathrm{C}$ with stirring, the solution was colorized to exhibit blue again. Indeed, the absorptions due to MB in the UV-Vis spectra gradually increased as shown in Figure 1, but the intensity was lower than that in the initial solution even after light shielding for $6 \mathrm{~h}$. The colorization was reasonably explained by the oxidation of MB caused by molecular oxygen. Furthermore, we confirmed that the change of the UV-Vis absorptions in the above experiments was similar as that observed in well-known redox reactions of MB by glucose and molecular oxygen in alkaline aqueous solution, which we had carried out as the comparative experiment according to the literature procedure [26].

Because it had been considered that the aforementioned reduction of $\mathrm{MB}$ in $\mathrm{BMIMCl}$ was probably owing to electron-transfer from BMIMCl to $\mathrm{MB}$ by the photo irradiation, evidence for occurrence of the electrontransfer was attempted to be provided by the fluorescence measurement of the solution of MB in BMIMCl. Figure 2 shows the fluorescence spectra of the solutions of $\mathrm{MB}$ in $\mathrm{BMIMCl}$ in various concentrations excited at 350 $\mathrm{nm}$, which is in the wavelength areas of the photo irradiation for the aforementioned reduction of MB. The emissions at around $450 \mathrm{~nm}$ due to BMIMCl decreased with increasing the concentrations of MB. The similar decrease of the emissions in the fluorescence spectra excited at other wavelengths such as 280,300 , and 330 $\mathrm{nm}$ was also observed with increasing the concentra- tions of $\mathrm{MB}$ in BMIMCl. Because MB had not exhibited the absorption at around $450 \mathrm{~nm}$ as shown in Figure 1, where the emission of BMIMCl was observed, however, it was evaluated that the decrease of the emissions in Figure 2

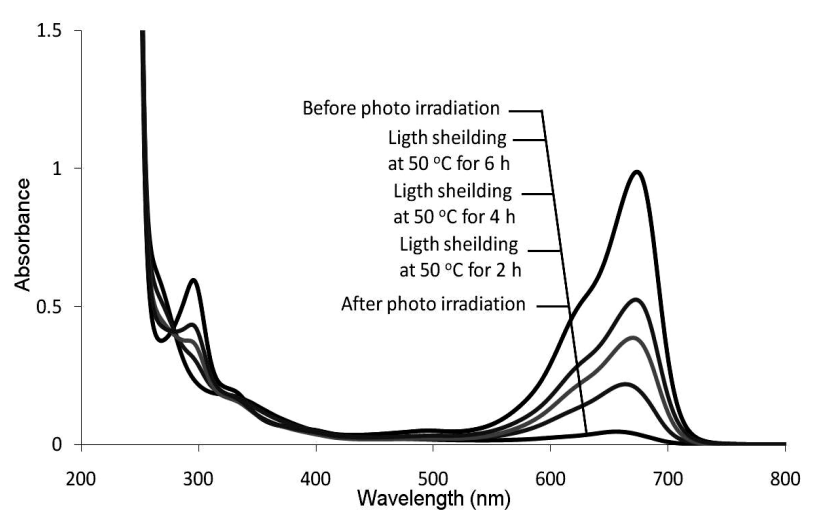

Figure 1. UV-Vis spectra of a solution of MB in BMIMCI before and after photo irradiation $(12 \mathrm{~h})$, and spectra of the solution which was left further standing under the conditions of light shielding at $50^{\circ} \mathrm{C}$ for 2,4 , and $6 \mathrm{~h}$.

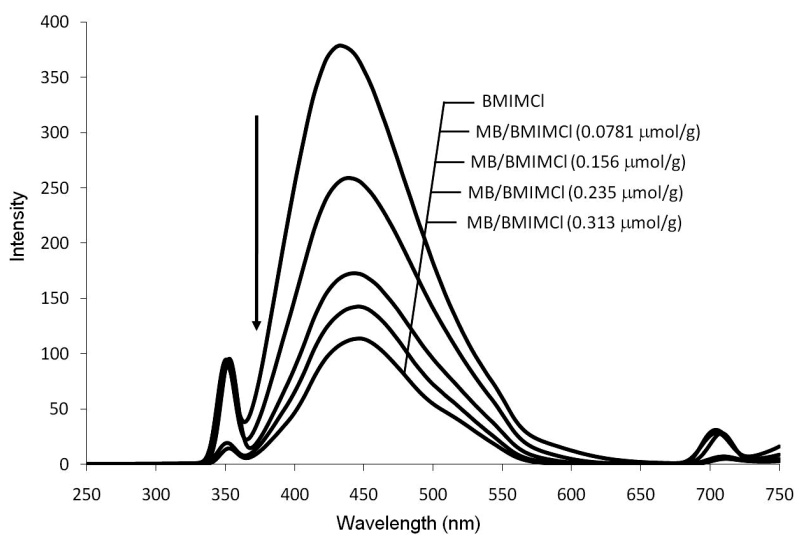

Figure 2. Fluorescence spectra of solutions of $\mathrm{MB}$ in $B M I M C l$ in various concentrations excited at $350 \mathrm{~nm}$.

was not attributed to energy-transfer from BMIMCl to MB by fluorescence resonance. Therefore, the result of the fluorescence measurement in Figure 2 can be taken to suggest that the electron-transfer has happened in the solution of $\mathrm{MB}$ in BMIMCl by the photo irradiation to cause the reduction of MB [27]. The similar excited state of an imidazolium-type ionic liquid was previously reported for the mechanism of photo-induced polymerization of aniline [28]. The aforementioned redox reactions of MB could be repeated by the cycle of the photo irradiation and light shielding experiments although the intensity of absorption at around $660 \mathrm{~nm}$ due to the oxidized state of MB in the UV-Vis spectra decreased according to the repeated numbers.

\section{Conclusions}

In this paper, we reported the photo-induced reduction of $\mathrm{MB}$ in BMIMCl. When photo irradiation at $280-370 \mathrm{~nm}$ wavelengths to the solution of $\mathrm{MB}$ in BMIMCl was carried out, decolorization of the solution occurred. The UVVis analysis of the solution suggested occurrence of the reduction of MB by the photo irradiation. Furthermore, the oxidation of MB by molecular oxygen took place by standing the solution under the conditions of light shielding at $50^{\circ} \mathrm{C}$. The fluorescence measurement of the solution excited at $350 \mathrm{~nm}$ indicated that the photo-induced reduction of MB probably happened via the electron-transfer from BMIMCl to MB. The detailed studies on the mechanism of the present reduction as well as the photo-induced reductions using other redox dyes in the ionic liquid are now in progress in our research group.

\section{Experimental}

All reagents were commercially available and used as received. Redox reaction of MB by glucose and molecu- 
lar oxygen in alkaline aqueous solution was conducted according to the literature procedure [26].

As a typical procedure for redox reactions of $\mathrm{MB}$ in BMIMCl, a solution of MB in BMIMCl $(0.125 \mu \mathrm{mol} / \mathrm{g})$ was placed in a quartz cuvette (thickness $1 \mathrm{~cm}$ ). After photo irradiation at $280-370 \mathrm{~nm}$ wavelengths (USHIO SX-UI 250HQ with 250W Hg lamp and UG11 filter) to the cuvette was performed at room temperature for $12 \mathrm{~h}$ for progress of the reduction, it was subjected to the UVVis measurement. Then, oxidation of MB was carried out by standing the solution under the conditions of light shielding at $50^{\circ} \mathrm{C}$ for $6 \mathrm{~h}$ with stirring, which was analyzed by the UV-Vis measurement. The UV-Vis and fluorescence spectra were recorded on Jasco V-650 spectrometer and FP-6300 fluorometer, respectively.

\section{References}

[1] A. Hulanicki and S. Glab, "Redox Indicators. Characteristics and Applications," Pure and Applied Chemistry, Vol. 50, No. 5, 1978, pp. 463-498. doi: $10.1351 / \mathrm{pac} 197850050463$

[2] S. B. Khoo and F. Chen, "Studies of Sol-Gel Ceramic Film Incorporating Methylene Blue on Glassy Carbon: An Electrocatalytic System for the Simultaneous Determination of Ascorbic and Uric Acids," Analytical Chemistry, Vol. 74, No. 22, 2002, pp. 5734-5741. doi:10.1021/ac0255882

[3] G. Zaitseva, Y. Gushikem, E. S. Ribeiro and S. S. Rosatto, "Electrochemical Property of Methylene Blue Redox Dye Immobilized on Porous Silica-Zirconia-Antimonia Mixed Oxide," Electrochimica Acta, Vol. 47, No. 9, 2002, pp. 1469-1474. doi:10.1016/S0013-4686(01)00870-2

[4] J.-Z. Xu, J.-J. Zhu, Q. Wu, Z. Hu and H.-Y. Chen, “An Amperometric Biosensor Based on the Coimmobilization of Horseradish Peroxidase and Methylene Blue on a Carbon Nanotubes Modified Electrode," Electroanalysis, Vol. 15, No. 3, 2003, pp. 219-224. doi:10.1002/elan.200390027

[5] Y. Yan, M. Zhang, K. Gong, L. Su, Z. Guo and L. Mao, "Adsorption of Methylene Blue Dye onto Carbon Nanotubes: A Route to an Electrochemically Functional Nanostructure and Its Layer-by-Layer Assembled Nanocomposite," Chemistry of Materials, Vol. 17, No. 13, 2005, pp. 3457-3463. doi:10.1021/cm0504182

[6] S. E. Salamifar, M. A. Mehrgardi, S. H. Kazemi and M. F. Mousavi, "Cyclic Voltammetry and Scanning Electrochemical Microscopy Studies of Methylene Blue Immbilized on the Self-assembled Monolayer of $n$-Dodecanethiol," Electrochimica Acta, Vol. 56, No. 2, 2010, pp. 896-904. doi:10.1016/j.electacta.2010.08.068

[7] S. Jain, G. Dangi, J. Vardia and S. C. Ameta, "Photocatalytic Reduction of Some Alkali Carbonates in the Presence of Methylene Blue," International Journal of Energy Research, Vol. 23, No. 1, 1999, pp. 71-77. doi:10.1002/(SICI)1099-114X(199901)23:1<71::AID-ER $\underline{464>3.0 . \mathrm{CO} ; 2-\mathrm{G}}$

[8] A. C. Borgo, A. M. Lazarin and Y. Gushikem, "Methylene Blue-Zirconium Phosphate-Cellulose Acetate Hybrid Membrane Film Attached to a Platinum Electrode and Its Application in Electrocatalytic Oxidation of NADH," Sensors and Actuators B: Chemical, Vol. 87, No. 3, 2002, pp. 498-505. doi:10.1016/S0925-4005(02)00291-5

[9] T. Welton, "Room-Temperature Ionic Liquids. Solvents for Synthesis and Catalysis," Chemical Reviews, Vol. 99, No. 8, 1999, pp. 2071-2084. doi:10.1021/cr980032t

[10] P. Wasserscheid and W. Keim, "Ionic Liquids-New 'Solutions' for Transition Metal Catalysis," Angewandte Chemie International Edition, Vol. 39, No. 21, 2000, pp. 3772-3789.

doi:10.1002/1521-3773(20001103)39:21<3772::AID-AN IE3772>3.0.CO;2-5

[11] D. Wei and A. Ivaska, "Applications of Ionic Liquids in Electrochemical Sensors," Analytica Chimica Acta, Vol. 607, No. 2, 2008, pp. 126-135. doi:10.1016/j.aca.2007.12.011

[12] T. Ueki and M. Watanabe, "Macromolecules in Ionic Liquids: Progress, Challenges, and Opportunities," Macromolecules, Vol. 41, No. 11, 2008, pp. 3739-3749. doi:10.1021/ma800171k

[13] A. Berthod, M. J. Ruiz-Ángel and S. Carda-Broch, "Ionic Liquids in Separation Techniques," Journal of Chromatography A, Vol. 1184, No. 1-2, 2008, pp. 6-18. doi:10.1016/j.chroma.2007.11.109

[14] A. Lewandowski and A. Swiderska-Mocek, "Ionic Liquids as Electrolytes for Li-Ion Batteries-An Overview of Electrochemical Studies," Journal of Power Sources, Vol. 194, No. 2, 2009, pp. 601-609. doi:10.1016/j.jpowsour.2009.06.089

[15] R. Giernoth, "Task-Specific Ionic Liquids," Angewandte Chemie International Edition, Vol. 49, No. 16, 2010, pp. 2834-2839.

[16] H. Izawa and J. Kadokawa, "Preparation and Characterizations of Functional Ionic Liquid-Gel and Hydrogel Materials of Xanthan Gum," Journal of Materials Chemistry, Vol. 20, No. 25, 2010, pp. 5235-5241. doi:10.1039/c0jm00595a

[17] S. Mine, K. Prasad, H. Izawa, K. Sonoda and J. Kadokawa, "Preparation of Guar Gum-Based Functional Materials Using Ionic Liquid," Journal of Materials Chemistry, Vol. 20, No. 41, 2010, pp. 9220-9225. doi:10.1039/c0jm00984a

[18] D. Seth, S. Sarkar, R. Pramanik, C. Ghatak, P. Setua and N. Sarkar, "Photophysical Studies of a Hemicyanine Dye (LDS-698) in Dioxane-Water Mixture, in Different Alcohols, and in a Room Temperature Ionic Liquid," The Journal of Physical Chemistry B, Vol. 113, No. 19, 2009, pp. 6826-6833. doi:10.1021/jp810045h

[19] C. Nese and A.-N. Unterreiner, "Photochemical Processes in Ionic Liquids on Ultrafast Timescales," Physical Chemistry Chemical Physics, Vol. 12, No. 8, 2010, pp. 1698-1708. doi:10.1039/b916799b

[20] H. Izawa, S. Wakizono and J. Kadokawa, "Fluorescence 
Resonance-Energy-Transfer in Systems of Rhodamine $6 \mathrm{G}$ with Ionic Liquid Showing Emissions by Excitation at Wide Wavelength Areas," Chemical Communications, Vol. 46, No. 34, 2010, pp. 6359-6361. doi:10.1039/c0cc01066a

[21] A. Paul and A. Samanta, "Photoinduced Electron Transfer Reaction in Room Temperature Ionic Liquids: A Combined Laser Flash Photolysis and Fluorescence Study," The Journal of Physical Chemistry B, Vol. 111, No. 8, 2007, pp. 1957-1962. doi:10.1021/jp067481e

[22] R. C. Vieira and D. E. Falvey, "Solvent-Mediated Photoinduced Electron Transfer in a Pyridinium Ionic Liquid," Journal of the Americal Chemical Society, Vol. 130, No. 5, 2008, pp. 1552-1553. doi:10.1021/ja077797f

[23] R. C. Vieira and D. E. Falvey, "Photoinduced ElectronTransfer Reactions in Two Room-Temperature Ionic Liquids: 1-Butyl-3-methylimidazolium Hexafluorophosphate and 1-Octyl-3-methylimidazolium Hexafluorophosphate," The Journal of Physical Chemistry B, Vol. 111, No. 18, 2007, pp. 5023-5029. doi:10.1021/jp0630471

[24] D. Behar, C. Gonzalez and P. Neta, "Reaction Kinetics in Ionic Liquids: Pulse Radiolysis Studies of 1-Butyl-3methylimidazolium Salts," The Journal of Physical Che- mistry A, Vol. 105, No. 32, 2001, pp. 7607-7614. doi:10.1021/jp011405o

[25] D. Behar, P. Neta and C. Schultheisz, "Reaction Kinetics in Ionic Liquids as Studied by Pulse Radiolysis: Redox Reactions in the Solvents Methyltributylammonium Bis (tri-fluoromethylsulfonyl)imide and $N$-Butylpyridinium Tetrafluoroborate," The Journal of Physical Chemistry A, Vol. 106, No. 13, 2002, pp. 3139-3147. doi:10.1021/jp013808u

[26] T. Lister, "Classic Chemistry Demonstrations: One Hundred Tried and Tested Experiments," Royal Society of Chemistry, London, 1995.

[27] K. V. Rao, K. Jayaramulu, T. K. Maji and S. J. George, "Supramolecular Hydrogels and High-Aspect-Ratio Nanofibers through Charge-Transfer-Induced Alternate Coassembly," Angewandte Chemie International Edition, Vol. 49, No. 25, 2010, pp. 4218-4222. doi:10.1002/anie. 201000527

[28] Z. Zhou, D. He, Y. Guo, Z. Cui, L. Zeng, G. Li and R. Yang, "Photo-Induced Polymerization in Ionic Liquid Medium: 1. Preparation of Polyaniline Nanoparticles," Polymer Bulletin, Vol. 62, No. 5, 2009, pp. 573-580. doi:10.1007/s00289-009-0038-y 